\title{
Checkpoint kinase 1 inhibitors as targeted molecular agents for clear cell carcinoma of the ovary (Review)
}

\author{
HIROSHI KOBAYASHI, HIROSHI SHIGETOMI and CHIHARU YOSHIMOTO \\ Department of Obstetrics and Gynecology, Nara Medical University, Kashihara, Nara 634-8522, Japan
}

Received August 18, 2014; Accepted April 29, 2015

DOI: 10.3892/ol.2015.3268

\begin{abstract}
In clear cell carcinoma of the ovary, chemoresistance frequently results in treatment failure. The present study aimed to review the potential association of transcription factor hepatocyte nuclear factor (HNF)-1 $\beta$ with cell cycle checkpoint machinery, as a mechanism for chemoresistance. The English-language literature on the subject was reviewed to identify genomic alterations and aberrant molecular pathways interacting with chemoresistance in clear cell carcinoma. Oxidative stress induced by repeated hemorrhage induces greater susceptibility of endometriotic cells to DNA damage, and subsequent malignant transformation results in endometriosis-associated ovarian cancer. Molecular changes, including those in HNF-1 $\beta$ and checkpoint kinase 1 (Chk1), may be a manifestation of essential alterations in cell cycle regulation, detoxification and chemoresistance in clear cell carcinoma. Chk1 is a critical signal transducer in the cell cycle checkpoint machinery. DNA damage, in turn, increases persistent phosphorylation of $\mathrm{Chk} 1$ and induction of $\mathrm{G} 2 / \mathrm{M}$ phase cell cycle arrest in cells overexpressing HNF-1 $\beta$. HNF-1 $\beta$ deletion induces apoptosis, suggesting that enhanced levels of HNF-1 $\beta$ may be associated with chemoresistance. Targeted therapy with Chk1 inhibitors may be explored as a potential treatment modality for patients with clear cell carcinoma. This provides a novel direction for combination therapy, including targeting of Chk1, which may overcome drug resistance and improve treatment efficacy.
\end{abstract}

\section{Contents}

1. Introduction

2. Materials and methods

Correspondence to: Professor Hiroshi Kobayashi, Department of Obstetrics and Gynecology, Nara Medical University, 840 Shijo-cho, Kashihara, Nara 634-8522, Japan

E-mail: hirokoba@naramed-u.ac.jp

Key words: chemoresistance, clear cell carcinoma, cell cycle checkpoint, targeted therapy
3. Iron-induced DNA damage in endometriosis and endometriosis-associated ovarian cancer

4. Molecular changes implicated in clear cell carcinoma

5. DNA damage response in clear cell carcinoma

6. Role of transcription factor HNF-1 $\beta$

7. Chk1 inhibitors as molecular targeted therapeutics

8. Conclusion

\section{Introduction}

Endometriosis is a common disease, which is associated with severe symptoms, including pelvic pain and infertility, and affects up to $10 \%$ of women of reproductive age $(1,2)$. Endometriosis also possesses a malignant transformation potential to ovarian carcinoma. Recently, several studies have indicated that heme and iron, present in endometriotic cysts, damage the endometriotic cells by inducing inflammation and oxidative stress (3-7). Simultaneously, several genes associated with acute antioxidant response, DNA damage repair and cell cycle modulation are activated. An increased understanding of the molecular biology involved in endometriosis-associated ovarian carcinogenesis may facilitate the identification of novel therapeutic targets.

The transcription factor hepatocyte nuclear factor (HNF)-1 $\beta$ is overexpressed in endometriosis and clear cell carcinoma of the ovary. HNF-1 $\beta$ may alleviate damage and promote the survival of cells experiencing stress, by upregulating antioxidant protein expression (8) and stimulating cell cycle checkpoint machinery (9). However, functional or clinical significance of this transcription factor is unclear. The present review aimed to summarize the potential association between HNF-1 $\beta$ and cell cycle checkpoint machinery.

The present review also outlines future directions for study, with respect to targeted therapy for ovarian clear cell carcinoma.

\section{Materials and methods}

A computerized literature search was conducted to identify relevant studies reported in the English language. MEDLINE electronic databases (http://www.ncbi.nlm.nih.gov/sites/entrez) published between 2000 and 2014 were searched, combining the keywords 'ovarian clear cell carcinoma', 'carcinogenesis, 'HNF-1 $\beta$ ' and 'DNA damage repair'. A variety of combinations 
of these terms were used, depending on which database was searched. Each gene was also linked to the relevant NCBI Entrez Gene pages (http://www.ncbi.nlm.nih.gov/sites/entrez). Furthermore, the references in each article were searched to identify potentially excluded studies.

\section{Iron-induced DNA damage in endometriosis and endo- metriosis-associated ovarian cancer}

Endometriosis is associated with hemorrhage or hemolysis. Repeated episodes of hemorrhage are a major cause of reproductive disability amongst patients with peritoneal endometriosis and endometriotic cysts (10). Erythrocytes existing outside the vascular system rapidly lyse and release free hemoglobin, and the blood scavenging system is required for effective hemoglobin clearance (11). Hemoglobin slowly undergoes spontaneous oxidation, also known as autoxidation (3), and is oxidized easily from the ferrous $\left(\mathrm{Fe}^{2+}\right)$ oxygenated form (oxyhemoglobin, $\left.\mathrm{HbO}_{2}\right)$ to the ferric $\left(\mathrm{Fe}^{3+}\right)$ met-form (methemoglobin, metHb) with generation of the superoxide anion $\left(\mathrm{O}_{2}^{-}\right)$as follows (4):

$$
\mathrm{HbO}_{2}^{-} \rightarrow \text { metHb }+\mathrm{O}_{2}^{-}
$$

Heme is also catabolized by heme oxygenases into iron, biliverdin and carbon monoxide. Free ferrous iron, in its catalytically active form, induces oxidative stress, if not chelated. This is due to the fact that $\mathrm{Fe}^{2+}$ catalyzes the non-enzymatic Fenton reaction (12), which produces highly reactive hydroxyl radicals as follows:

$$
\mathrm{Fe}^{2+}+\mathrm{H}_{2} \mathrm{O}_{2}^{-} \rightarrow \mathrm{Fe}^{3+}+\mathrm{HO}^{-}+\cdot \cdot \mathrm{OH}
$$

$\mathrm{Fe}^{2+}$ is involved in the formation of reactive oxygen species (ROS) and reactive nitrogen species (RNS), which are damaging to the majority of cell components, including DNA, membranes and proteins, and induce cell death. The hydroxyl radical $(\bullet \mathrm{OH})$ and superoxide anion $\left(\mathrm{O}_{2}^{-}\right)$are toxic to living organisms. 8-hydroxy-2'-deoxyguanosine (8-OxodG) is a major form of oxidative DNA, generated following exposure to free radicals, and is released from cells following DNA repair (13). A study indicated that the levels of 8-OxodG in peritoneal fluid were higher in women with endometriosis compared with those of the control groups (5). The repeated hemorrhage within the endometriotic lesions, hemoglobin breakdown and subsequent heme and iron accumulation mediate secondary tissue injury and result in cell death (14). Therefore, dead cells are unable to induce ovarian carcinogenesis.

Paradoxically, it is generally accepted that persistent oxidative stress may be involved in chronic inflammation, which in turn, may mediate chronic diseases including cancer (6). Several studies reported that continued oxidative stress leads not only to increased cell survival, but also increased tumorigenic potential of cancer cells $(6,7)$. Oxidative stress contributes to the expansion of genomic instability throughout the genome, and subsequent gene mutations involved in the initiation of tumorigenesis (15). In an oxidative stress situation, iron overload-associated diseases, including hemochromatosis, chronic viral hepatitis, asbestosis and endometriosis, may result in the development of neoplasia, including hepatocellular carcinoma, malignant mesothelioma and ovarian cancer (16). Such microenvironments share common mechanistic foundations or provide a biological interface between cell death and carcinogenesis induced by persistent oxidative stress.
Antioxidants are key regulators in the maintenance of cellular redox balance and inhibition of the susceptibility to tumorigenesis. Animal experiments revealed that loss of ROS-scavenging enzymes, for example peroxiredoxin, was associated with an increase in cancer susceptibility (17). By contrast, the overexpression of antioxidants, including $\mathrm{NAD}(\mathrm{P}) \mathrm{H}$ : quinone oxidoreductase 1 (NQO1), has been correlated with numerous human malignancies, suggesting a role in carcinogenesis (18). The endometriosis-specific transcription factor, HNF-1 $\beta$, functions to alleviate endometriotic cell damage and also promotes survival of cells under oxidative stress by upregulating antioxidant protein expression (8). It was hypothesized that this increase in antioxidants may reduce the susceptibility of endometriotic cells to lethal injury from ROS, following exposure to heme and iron. Therefore, overexpression of antioxidant enzymes may, in part, be responsible for the increased risk of cancer amongst women with endometriosis. Excess ROS induce significant oxidative stress, resulting in cell death, whereas sublethal ROS levels may adapt to prolong cell survival and increase the tumorigenic potential of endometriotic cells. Each endometriotic lesion may display differences with regards to the level of responsiveness to ROS. Carcinogenesis requires a delicate balance between oxidants and antioxidants.

\section{Molecular changes implicated in clear cell carcinoma}

Endometriosis was suggested to confer an elevated risk of epithelial ovarian cancer, particularly the clear cell and endometrioid adenocarcinoma subtypes, also known as endometriosis-associated ovarian cancer (EAOC) $(1,2)$. Clear cell carcinoma and endometrioid adenocarcinoma exhibit distinct clinicopathological features and molecular phenotypes (19,20). Yamada et al (21) hypothesized that heme and iron-induced signals contribute to carcinogenesis via three major mechanisms: i) By enhancing oxidative stress, which promotes DNA mutagenesis and contributes to tumor initiation; ii) via activation of detoxification pathways, thereby contributing to tumor promotion; iii) by sustaining growth of cancer cells via estrogen-dependent (endometrioid) or -independent (clear cell) mechanisms. These studies provide novel insights into the pathophysiology and significance of genetic functional categories in EAOC. Several molecular changes that have been implicated in the clear cell carcinoma pathway include: i) Chemoresistance (22-26); ii) cell cycle regulation; iii) detoxification; iv) hormone independency; v) chromosomal instability; and vi) glycogen synthesis (24).

Clear cell carcinoma is more likely to exhibit chemoresistance than high-grade serous carcinoma, demonstrating that clear cell histology is highly resistant to conventional platinum-based chemotherapy (23). Potential doubling time for clear cell carcinoma cells was longer than that of serous adenocarcinoma cells (61 vs. $30 \mathrm{~h}$ ), suggesting that the intrinsic chemoresistance may be associated with prolonged doubling time (22). Decreased proliferation rates of cancer cells promotes resistance to a number of chemotherapeutics; a behavior observed in clear cell carcinoma. This chemoresistance may also be associated with an increase in cell cycle arrest during the DNA damage response, or enhanced drug detoxification within the cell (27). The cell cycle remains arrested 
to repair DNA damage induced by heme and iron-induced oxidative stress (see the 'Role of transcription factor HNF-1 $\beta$ ' section for details). Genetic and epigenetic dysregulation of chemoresistance-associated genes may induce cell cycle arrest and suppress cell proliferation.

The pathogenesis of endometriosis-associated ovarian carcinogenesis may be closely associated with heme and iron overload and the associated overexpression of antioxidants (27). Redox-sensitive antioxidant genes may be associated with the downstream targets of HNF-1 $\beta(27,28)$. HNF-1 $\beta$ mainly remains epigenetically hypomethylated and enhances ROS detoxification, resulting in typically low ROS levels in clear cell carcinoma $(8,29)$. Therefore, it is conceivable that excess ROS induces cell death, while sublethal doses of oxidative stress may accelerate the development and progression of clear cell carcinogenesis.

Clear cell and endometrioid carcinoma are genetically distinct cancers, and whilst clear cell carcinoma presents rare expression of the estrogen receptor (ER) and progesterone receptor $(\mathrm{PR})$, the $\mathrm{ER}$ and $\mathrm{PR}$ are often overexpressed in endometrioid carcinoma. Endometrioid adenocarcinomas are more likely to co-express ER and PR compared with the other histological subtypes (30). By contrast, clear cell carcinomas are characterized by their negative expression of hormone receptors (30).

The most conspicuous feature of clear cell carcinoma is the abundance of intracellular glycogen (31). The HNF-1 $\beta$ signature is associated with glycogen metabolism, including that of glucose-6-phosphatase (32). Hypoxia induces a switch from oxidative phosphorylation to glycolysis, increasing glycogen synthesis (31). Hypoxia inducible factor (HIF)-1 $\alpha$ overexpression in clear cell carcinoma resulted in an increase in the production of glycogen synthase 1 (GYS1), indicating that a hypoxic environment promoted glycogen synthesis. Taken together, these results indicated that HNF-1 $\beta$ overexpression in clear cell carcinoma has a role in glycogen synthesis, cell cycle regulation and detoxification.

\section{DNA damage response in clear cell carcinoma}

The genome is constantly damaged by endogenous compounds, for example ROS resulting from metabolic processes, or exogenous compounds, including ionizing radiation, ultraviolet and environmental toxins. Oxidative stress, chronic injury and inflammation promote DNA damage and chromosomal aberrations, which are processed by series of pathways known as the 'DNA damage response (DDR)'. The DDR pathways regulate cell fate decisions regarding DNA repair, cell cycle arrest and ultimately cell death, senescence or survival. The DDR represents a complex network of signaling pathways, which halt the cell cycle to allow time for DNA repair. DDR pathways coordinate multiple repair processes, including nucleotide excision repair (NER), base excision repair (BER), mismatch repair (MMR), DNA double strand break (DSB) repair and post replication repair (PRR), facilitating the maintenance of genomic integrity following exposure to various stressors (33).

Individual DNA base damage and DNA intrastrand crosslinks are removed by the processes of BER and NER, respectively. The BER process constitutes the primary defense mechanism exerted by DNA glycosylase enzymes, 8-oxoguanine DNA glycosylase (OGG1) and X-ray repair complementing defective repair in Chinese hamster cells 1 . The NER pathway repairs DNA damage induced by UV light and oxidative stress (34). Furthermore, cellular sensing machinery for DNA damage includes: i) Poly(ADP-ribose) polymerase 1 ; ii) members of the phosphatidylinositol 3-kinase protein family known as ataxia-telangiectasia mutated (ATM), ataxia-telangiectasia and Rad3-related (ATR); iii) checkpoint kinases 1 and 2 (Chk1 and Chk2); iv) the dual-specificity protein phosphatases CDC25A-C; and v) cyclin-dependent kinases (CDK2/4) (35). Generally, single-strand DNA initiates ATR-Chk1 pathway activation, whereas DSBs promote ATM-Chk2 activation. DSBs are repaired by non-homologous end joining or by homologous recombination. Chk1, an important signal transducer in the cell cycle checkpoint pathway, represents a core component, which contributes to all cell cycle checkpoints (36).

Recent studies have demonstrated the chemoresistance mechanism in clear cell carcinoma (37). Among ovarian cancer, excision repair cross-complementation group 1 (ERCC1) and ERCC3 are specifically overexpressed in clear cell carcinoma (38). The AT rich interactive domain 1A, SWI-like (ARID1A) is a chromatin remodeling gene frequently mutated in a variety of female reproductive organ-derived cancers, including clear cell carcinoma (39). Thus, the cell cycle checkpoint machinery has become a focus of research in the field of anticancer drug resistance. Genome-wide and proteome-based approaches provide crucial information regarding the target molecules that modulate the cell cycle checkpoint pathways. The HNF-1 $\beta$ gene is one of the key molecules associated with the ATR-Chk1 pathway.

\section{Role of transcription factor HNF-1及}

Hypomethylation and increased protein levels of HNF-1 $\beta$ are specific features of endometriosis and its malignant transformation into clear cell carcinoma of the ovary. The DNA repair capacity of a cell contributes to its genomic integrity and is vital to the normal functioning of an organism. A recent study reported that a molecular link between ATR and Chk1 was significant in the cell cycle regulatory pathway in clear cell carcinoma (9). Transient phosphorylation of Chk1 is critical for successful recovery of the cell cycle following stalled DNA replication (9). This section summarizes recent progress in the elucidation of transcription factor HNF-1 $\beta$-mediated regulation of DNA damage-induced cell cycle arrest.

As shown in Fig. 1, HNF-1 $\beta$ is critical to the anti-oxidative effects observed in endometriosis and clear cell carcinoma (27). Furthermore, this transcription factor inhibits apoptosis of cancer cells. However, the precise mechanism of HNF-1 $\beta$ action is yet to be fully elucidated. Shigetomi et al (9) confirmed that HNF-1 $\beta$ induced $\mathrm{G} 2 / \mathrm{M}$ phase arrest and anti-apoptosis in clear cell carcinoma cells via an enhancement of the oxidative DNA damage-induced ATR-Chk1-associated pathway. Blockade of this event by HNF-1 $\beta$ short interfering (si)RNA induced apoptosis and an impaired G2/M checkpoint following exposure to oxidative stress $(9,40)$. Notably, HNF-1 $\beta$ performs a critical role in DDR by controlling cell cycle regulation via activation of the ATR-Chk1 pathway. These results provide novel insights into the critical roles of HNF-1 $\beta$ in the 


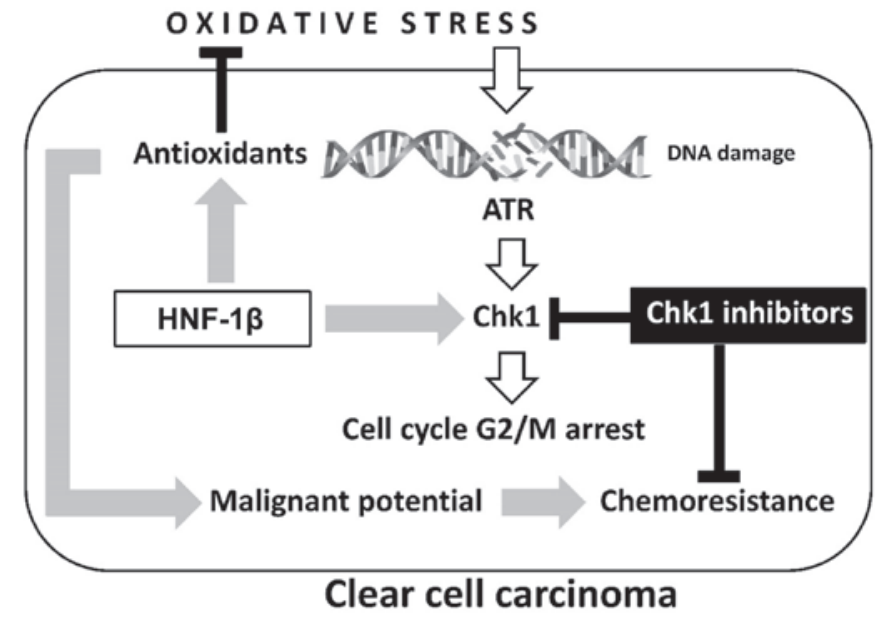

Figure 1. Two major hallmarks, oxidative stress and antioxidants, are crucial in the development of ovarian clear cell carcinoma: Oxidative stress contributes to immediate phosphorylation of Chk1 by ATR in the presence of DNA damage. HNF-1 $\beta$ overexpression induces antioxidant expression and persistent Chk1 activation, which are associated with detoxification and cell cycle arrest, respectively. The chemoresistance of clear cell carcinoma may be due to aberrant retention of the $\mathrm{G} 2$ checkpoint through overexpression of $\mathrm{HNF}$ 1ß. Oxidative stress-mediated excess ROS induces cell death, while sublethal ROS may adapt to prolonged cell survival and increase the tumorigenic potential of endometriotic cells. Carcinogenesis requires a balance between oxidants and antioxidants. Collectively, our data demonstrate the importance of HNF-1 $\beta$ as a promising treatment strategy for overcoming chemoresistance in clear cell carcinoma. HNF-1 $\beta$, hepatocyte nuclear factor-1 $\beta$; ROS reactive oxygen species; Chk1, checkpoint 1; ATR, ataxia-telangiectasia and Rad3-related.

regulation of oxidative stress-induced G2/M cell cycle arrest and anti-apoptosis in clear cell carcinoma cells.

On one hand, cell cycle arrest provides pre-malignant endometriotic cells with the opportunity to persistently repair DNA following oxidative damage. However, on the other hand, HNF-1 $\beta$ induces an increase in G2/M accumulation and decrease in the mitotic entry of DNA-damaged cells and apoptosis. These cells fail to maintain genomic integrity and may enhance malignant potential. Several reports have indicated that elevated levels of HNF-1 $\beta$ are associated with chemoresistance, which may be suppressed by HNF-1 $\beta$ siRNA expression $(9,40)$. Targeting Chk1 also blocks HNF-1 $\beta$-induced Chk1 phosphorylation and markedly increases lethality in vitro. These data led to the hypothesis that combining checkpoint inhibitors with DNA damaging agents may force cancer cells into premature and lethal mitosis, via an accumulation of genomic instability by overriding checkpoints. The role of HNF-1 $\beta$ in DDR through the ATR-Chk1 pathway, and potential combinatorial treatment strategies will be discussed with a mechanistic rationale.

\section{Chk1 inhibitors as molecular targeted therapeutics}

The potential implications of the aforementioned factors in therapeutic intervention were subsequently evaluated. Integration of gene expression profiling and proteomics analysis to identify genomic alterations and aberrant molecular pathways in a clinical setting is necessary to facilitate personalized targeted therapy. If predictive biomarkers are identified, the use of targeted agents may improve prognosis.
DNA damage activates DDR and the DNA repair pathway, including Chk1. Chk1 is a serine-threonine checkpoint kinase central to the DDR network, which represents one of the most important targets for anti-cancer therapeutics. In preclinical studies, Chk1 inhibition enhanced the cytotoxicity of several chemotherapeutic agents (9). UCN-01 (7-hydroxystaurosporine), the first non-selective Chk1 inhibitor introduced in a clinical study, combined with irinotecan demonstrated activity in patients with advanced triple-negative breast cancer (41). In the phase II clinical study, however, this regimen induced limited activity in triple-negative breast cancer (41). Although Chk1 inhibitors, for example AZD7762 and PF-477736, also failed to induce anti-cancer effects as significant as those exerted by single agents, they have the potential, as chemosensitizers, to sensitize cancer cells to a variety of DNA-damaging agents. AZD7762 is an adenosine triphosphate (ATP)-competitive Chk1/2 inhibitor. PF-477736 is a potent, selective ATP-competitive Chk1 inhibitor. Phase I/II clinical studies revealed that Chk1 inhibitors exhibited limited activity, which often outweighed the marginal efficacy benefit in combination with chemotherapy in certain types of cancer, including advanced solid cancer and refractory acute leukemia $(41,42)$. Unfortunately, there have as yet been no reports in the literature of profound enhancements in patient survival.

Two major factors, which have crucial roles in the development of clear cell carcinoma, include HNF-1 $\beta$ overexpression and persistent Chk1 activation, associated with detoxification and cell cycle arrest, respectively (Fig. 1). Chemoresistance may be regulated by HNF-1 $\beta$-dependent continued activation of Chk1 (9). Accurate assessment of the overexpression of HNF-1 $\beta$ and continued activation of Chk1 in clear cell carcinoma tissues aid the identification of personalized Chk1 therapies to molecularly selected patients. Therefore, Chk1 inhibitors will be approved for selected patients whose HNF-1 $\beta$ and Chk1 status has been defined by immunohistochemical analysis. Such future personalized therapeutic approaches warrant further research in vivo, via animal studies and clinical trials.

\section{Conclusion}

The present study reviews current knowledge regarding the epigenetic and genetic alterations, and aberrant molecular pathways underlying clear cell carcinoma, as well as discussing the advancement of personalized therapeutic approaches. Furthermore, the association of HNF-1 $\beta$ with cell cycle checkpoint machinery was also evaluated as a mechanism for chemoresistance.

Clear cell carcinoma develops high intrinsic resistance to conventional platinum-based chemotherapy (43). Genome-wide profiling analyses and proteomic-based studies revealed that genes associated with oxidative stress, detoxification and chemoresistance were enriched in clear cell carcinoma $(21,24-27)$. These analyses identified the molecular basis of diverse biological phenomena mediated by transcription factor HNF-1 $\beta$, and suggest that HNF-1 $\beta$ contributes to the enhancement of cell cycle checkpoint machinery against oxidative stress-induced DNA damage. Clear cell carcinoma-specific HNF-1 $\beta$ is vital for proper 
cell cycle G2/M phase arrest and induces chemoresistance. HNF-1 $\beta$ siRNA treatment abrogates cell cycle arrest and results in apoptosis in HNF-1 $\beta$-overexpressing clear cell carcinoma cells (40). The loss of HNF-1 $\beta$ may also enhance chemosensitivity (9). Therefore, pharmacological inactivation of HNF-1 $\beta$ is an emerging concept underlying the development of novel anti-cancer agents. However, since the HNF-1 $\beta$ gene is expressed in important organs, including the liver and kidney, this targeted therapy may induce significant adverse reactions.

The progression of clear cell carcinoma is driven by specific genomic alterations, including hypomethylation of the HNF-1 $\beta$ gene, leading to the generation of abnormal proteins (continued Chk1 phosphorylation) that can be targeted. Based on recent observations, targeting HNF-1 $\beta$-associated downstream pathways, for example ATR-Chk1 signaling, may mediate tumoricidal effects (9). The ATR kinase serves as a transducer of the oxidative stress-dependent damage signal, phosphorylating and activating the downstream effector kinase Chk1. Chk1 inhibitors inhibit the growth of sensitive HNF-1 $\beta$-overexpressing cells by inducing significant levels of cell apoptosis, in addition to contributing to therapeutic efficacy of HNF-1 $\beta$-based treatments $(41,42)$. The effectiveness of Chk1 inhibitors may be dependent on HNF-1 $\beta$ overexpression in clear cell carcinoma. Therefore, target-based agents are active only in molecularly selected populations of patients. The combination of conventional chemotherapy and targeted Chk1-inhibitor therapy may be explored as a personalized treatment modality for selected patients presenting tumors with HNF-1 $\beta$ overexpression. HNF-1 $\beta$ and Chk1 may also represent candidates for novel therapeutic targets in clear cell carcinoma.

In conclusion, the present review provides a possible explanation for why clear cell carcinoma still possesses the cell cycle arrest response, and discusses current knowledge regarding how this type of cancer affects the ATR-Chk1 network. Predictive biomarkers of ATR-Chk1 network activity may indicate the specific subgroup of patients able to benefit from Chk1 inhibitors.

\section{Acknowledgements}

The present review was supported by a grant-in-aid for Scientific Research from the Ministry of Education, Science and Culture of Japan (no. 26293361) to the Department of Obstetrics and Gynecology, Nara Medical University (to Professor Hiroshi Kobayashi).

\section{References}

1. Bulun SE: Endometriosis. N Engl J Med 360: 268-279, 2009.

2. Dzatic-Smiljkovic O, Vasiljevic M, Djukic M, Vugdelic R and Vugdelic J: Frequency of ovarian endometriosis in epithelial ovarian cancer patients. Clin Exp Obstet Gynecol 38: 394-398, 2011.

3. Abugo OO and Rifkind JM: Oxidation of hemoglobin and the enhancement produced by nitroblue tetrazolium. J Biol Chem 269: 24845-24853, 1994.

4. Shikama K and Matsuoka A: Human haemoglobin: A new paradigm for oxygen binding involving two types of alphabeta contacts. Eur J Biochem 270: 4041-4051, 2003

5. Polak G, Wertel I, Barczyński B, Kwaśniewski W, Bednarek W and Kotarski J: Increased levels of oxidative stress markers in the peritoneal fluid of women with endometriosis. Eur J Obstet Gynecol Reprod Biol 168: 187-190, 2013.
6. Reuter S, Gupta SC, Chaturvedi MM and Aggarwal BB: Oxidative stress, inflammation, and cancer: How are they linked? Free Radic Biol Med 49: 1603-1616, 2010.

7. Mahalingaiah PK and Singh KP: Chronic oxidative stress increases growth and tumorigenic potential of MCF-7 breast cancer cells. PLoS One 9: e87371, 2014.

8. Akasaka J, Uekuri C, Shigetomi H, Koike M and Kobayashi H: Hepatocyte nuclear factor (HNF)- $1 \beta$ and its physiological importance in endometriosis. Biomed Rep 1: 13-17, 2013.

9. Shigetomi H, Sudo T, Shimada K, Uekuri C, Tsuji Y, Kanayama S, Naruse K, Yamada Y, Konishi N and Kobayashi H: Inhibition of cell death and induction of G2 arrest accumulation in human ovarian clear cells by HNF-1 $\beta$ transcription factor: Chemosensitivity is regulated by checkpoint kinase CHK1. Int J Gynecol Cancer 24: 838-843, 2014.

10. Kobayashi H, Kajihara H, Yamada Y, Tanase Y, Kanayama S, Furukawa N, Noguchi T, Haruta S, Yoshida S, Naruse K, et al: Risk of carcinoma in women with ovarian endometrioma. Front Biosci (Elite Ed) 3: 529-539, 2011.

11. Liu C, Liu X, Janes J, Stapley R, Patel RP, Gladwin MT and Kim-Shapiro DB: Mechanism of faster NO scavenging by older stored red blood cells. Redox Biol 2: 211-219, 2014.

12. Cadet J, Delatour T, Douki T, Gasparutto D, Pouget JP, Ravanat JL and Sauvaigo S: Hydroxyl radicals and DNA base damage. Mutat Res 424: 9-21, 1999.

13. Wei H, Cai Q and Rahn RO: Inhibition of UV light- and Fenton reaction-induced oxidative DNA damage by the soybean isoflavone genistein. Carcinogenesis 17: 73-77, 1996.

14. Kobayashi H, Kajiwara H, Kanayama S, Yamada Y, Furukawa N, Noguchi T, Haruta S, Yoshida S, Sakata M, Sado T and Oi H: Molecular pathogenesis of endometriosisassociated clear cell carcinoma of the ovary (review). Oncol Rep 22: 233-240, 2009.

15. Xue X and Shah YM: Intestinal iron homeostasis and colon tumorigenesis. Nutrients 5: 2333-2351, 2013.

16. Toyokuni S: Mysterious link between iron overload and CDKN2A/2B. J Clin Biochem Nutr 48: 46-49, 2011.

17. Rolfs F, Huber M, Gruber F, Böhm F, Pfister HJ, Bochkov VN, Tschachler E, Dummer R, Hohl D, Schäfer M, et al: Dual role of the antioxidant enzyme peroxiredoxin 6 in skin carcinogenesis. Cancer Res 73: 3460-3469, 2013

18. Yang Y, Zhang Y, Wu Q, Cui X, Lin Z, Liu S and Chen L: Clinical implications of high NQO1 expression in breast cancers. J Exp Clin Cancer Res 33: 14, 2014.

19. Tanase Y, Yamada Y, Shigetomi H, Kajihara H, Oonogi A, Yoshizawa Y, Furukawa N, Haruta S, Yoshida S, Sado T, et al: Modulation of estrogenic action in clear cell carcinoma of the ovary (Review). Exp Ther Med 3: 18-24, 2012.

20. Kajihara H, Yamada Y, Shigetomi H, Higashiura Y and Kobayashi H: The dichotomy in the histogenesis of endometriosis-associated ovarian cancer: Clear cell-type versus endometrioid-type adenocarcinoma. Int J Gynecol Pathol 31: 304-312, 2012.

21. Yamada Y, Shigetomi H, Onogi A, Haruta S, Kawaguchi R, Yoshida S, Furukawa N, Nagai A, Tanase Y, Tsunemi T, et al: Redox-active iron-induced oxidative stress in the pathogenesis of clear cell carcinoma of the ovary. Int J Gynecol Cancer 21: 1200-1207, 2011.

22. Itamochi H, Kigawa J, Akeshima R, Sato S, Kamazawa $S$, Takahashi M, Kanamori Y, Suzuki M, Ohwada M and Terakawa N: Mechanisms of cisplatin resistance in clear cell carcinoma of the ovary. Oncology 62: 349-353, 2002.

23. Pectasides D, Pectasides E, Psyrri A and Economopoulos T: Treatment issues in clear cell carcinoma of the ovary: A different entity? Oncologist 11: 1089-1094, 2006.

24. Yoshida S, Furukawa N, Haruta S, Tanase Y, Kanayama S, Noguchi T, Sakata M, Yamada Y, Oi H and Kobayashi H: Theoretical model of treatment strategies for clear cell carcinoma of the ovary: Focus on perspectives. Cancer Treat Rev 35: 608-615, 2009.

25. Mogami T, Yokota N, Asai-Sato M, Yamada R, Koizume S, Sakuma Y, Yoshihara M, Nakamura Y, Takano Y, Hirahara F, et al: Annexin A4 is involved in proliferation, chemo-resistance and migration and invasion in ovarian clear cell adenocarcinoma cells. PLoS One 8: e80359, 2013.

26. Tsuda H, Ito YM, Ohashi Y, Wong KK, Hashiguchi Y, Welch WR, Berkowitz RS, Birrer MJ and Mok SC: Identification of overexpression and amplification of $\mathrm{ABCF} 2$ in clear cell ovarian adenocarcinomas by cDNA microarray analyses. Clin Cancer Res 11: 6880-6888, 2005. 
27. Kajihara H, Yamada Y, Kanayama S, Furukawa N, Noguchi T, Haruta S, Yoshida S, Sado T, Oi H and Kobayashi H: Clear cell carcinoma of the ovary: Potential pathogenic mechanisms (Review). Oncol Rep 23: 1193-1203, 2010.

28. Mandai M, Matsumura N, Baba T, Yamaguchi K, Hamanishi J and Konishi I: Ovarian clear cell carcinoma as a stress-responsive cancer: Influence of the microenvironment on the carcinogenesis and cancer phenotype. Cancer Lett 310: 129-133, 2011.

29. Yamaguchi K, Huang Z, Matsumura N, Mandai M, Okamoto T, Baba T, Konishi I, Berchuck A and Murphy SK: Epigenetic determinants of ovarian clear cell carcinoma biology. Int J Cancer 135 585-597, 2014.

30. Hecht JL, Kotsopoulos J, Hankinson SE and Tworoger SS Relationship between epidemiologic risk factors and hormone receptor expression in ovarian cancer: Results from the Nurses Health Study. Cancer Epidemiol Biomarkers Prev 18: 1624-1630, 2009.

31. Iida Y, Aoki K, Asakura T, Ueda K, Yanaihara N, Takakura S, Yamada K, Okamoto A, Tanaka T and Ohkawa K: Hypoxia promotes glycogen synthesis and accumulation in human ovarian clear cell carcinoma. Int J Oncol 40: 2122-2130, 2012.

32. Cuff J, Salari K, Clarke N, Esheba GE, Forster AD, Huang S, West RB, Higgins JP, Longacre TA and Pollack JR: Integrative bioinformatics links HNF1B with clear cell carcinoma and tumor-associated thrombosis. PLoS One 8: e74562, 2013.

33. Dai Y and Grant S: New insights into checkpoint kinase 1 in the DNA damage response signaling network. Clin Cancer Res 16: 376-383, 2010.

34. Zharkov DO: Base excision DNA repair. Cell Mol Life Sci 65: $1544-1565,2008$

35. Smith J, Tho LM, Xu N and Gillespie DA: The ATM-Chk2 and ATR-Chk1 pathways in DNA damage signaling and cancer. Adv Cancer Res 108: 73-112, 2010.

36. Reinhardt $\mathrm{HC}$ and Yaffe MB: Kinases that control the cell cycle in response to DNA damage: Chk1, Chk2, and MK2. Curr Opin Cell Biol 21: 245-255, 2009.
37. Sugiyama T, Kamura T, Kigawa J, Terakawa N, Kikuchi Y, Kita T, Suzuki M, Sato I and Taguchi K: Clinical characteristics of clear cell carcinoma of the ovary: A distinct histologic type with poor prognosis and resistance to platinum-based chemotherapy. Cancer 88: 2584-2589, 2000.

38. Reed E, Yu JJ, Davies A, Gannon J and Armentrout SL: Clear cell tumors have higher mRNA levels of ERCC1 and XPB than other histological types of epithelial ovarian cancer. Clin Cancer Res 9: 5299-5305, 2003.

39. Mao TL and Shih IeM: The roles of ARID1A in gynecologic cancer. J Gynecol Oncol 24: 376-381, 2013.

40. Tsuchiya A, Sakamoto M, Yasuda J, Chuma M, Ohta T, Ohki M, Yasugi T, Taketani Y and Hirohashi S: Expression profiling in ovarian clear cell carcinoma: Identification of hepatocyte nuclear factor- 1 beta as a molecular marker and a possible molecular target for therapy of ovarian clear cell carcinoma. Am J Pathol 163: 2503-2512, 2003.

41. Ma CX, Ellis MJ, Petroni GR, Guo Z, Cai SR, Ryan CE, Lockhart AC, Naughton MJ, Pluard TJ, et al: A phase II study of UCN-01 in combination with irinotecan in patients with metastatic triple negative breast cancer. Breast Cancer Res Treat 137: 483-492, 2013.

42. Kaufmann SH, Karp JE, Litzow MR, Mesa RA, Hogan W, Steensma DP, Flatten KS, Loegering DA, Schneider PA, Peterson KL, et al: Phase I and pharmacological study of cytarabine and tanespimycin in relapsed and refractory acute leukemia. Haematologica 96: 1619-1626, 2011.

43. Takano M, Kikuchi Y, Yaegashi N, Kuzuya K, Ueki M, Tsuda H, Suzuki M, Kigawa J, Takeuchi S, Tsuda H, et al: Clear cell carcinoma of the ovary: A retrospective multicentre experience of 254 patients with complete surgical staging. Br J Cancer 94: 1369-1374, 2006. 